\title{
仲胺介入的丙炔酸甲酯与亚胺的环合反应
}

\author{
马鸿飞 ${ }^{a}$ 黄 浩 ${ }^{a}$ 宿江岭 ${ }^{b}$ 钮长盛 ${ }^{a}$ \\ 吴正光 ${ }^{c} \quad$ 卜洪忠 ${ }^{a}$ 李玉峰*, $a$ \\ ( ${ }^{a}$ 南京工业大学化学与分子工程学院 南京 211816) \\ ( ${ }^{b}$ 山东万博化工有限公司 惱博 255000) \\ ( ${ }^{c}$ 南京大学化学化工学院 南京 210093)
}

\begin{abstract}
摘要 建立了一种合成四氢嘧啶衍生物的方法. 仲胺与丙炔酸甲酯发生 Michael 加成反应, 生成 $\beta$-胺基丙烯酸酯; 向 $\beta$ 胺基丙烯酸甲酯及亚胺的混合物中加入有机酸, 常温下数分钟内发生环合反应得到四氢嘧啶衍生物, 仲胺以铵盐形式 离去. 该反应对甲酫亚胺具有良好的适应性.
\end{abstract}

关键词＼cjkstart丙炔酸甲酯; 亚胺; 胺介入反应; 四氢嘧啶衍生物

\section{Secondary Amine-Mediated Cyclization of Methyl Propiolate with Imines}

\author{
Ma, Hongfei ${ }^{a} \quad{\text { Huang, } \mathrm{Hao}^{a} \quad \mathrm{Su} \text {, Jiangling }}^{b} \quad$ Niu, Changsheng ${ }^{a}$ \\ $\mathrm{Wu}$, Zhengguang ${ }^{c} \quad \mathrm{Bu}$, Hongzhong ${ }^{a} \quad \mathrm{Li}$, Yufeng ${ }^{*, a}$ \\ $\left({ }^{a}\right.$ College of Chemistry and Molecular Engineering, Nanjing Tech University, Nanjing 211816) \\ $\left({ }^{b}\right.$ Shandong Wanbo Chemical Industry Corporation, Zibo 255000) \\ ( ${ }^{c}$ Chemistry and Chemical Engineering, Nanjing University, Nanjing 210093)
}

\begin{abstract}
A facile method for the synthesis of tetrahydropyrimidines is described. Diethylamine was treated with methyl propiolate to provide Michael adduct ( $\beta$-amino acrylate). In the presence of organic acid, the cyclization between imines with $\beta$-aminoacrylateoccurred at room temperature and completed within several minutes to give tetrahydropyrimidines with liberation of ammonium salt. Formaldimines show good toleration to the reaction.
\end{abstract}

Keywords methylpropiolate; imine; amine-mediated reaction; tetrahydropyrimidines

众所周知, 胺催化在当代有机合成中扮演了很重要 的角色. 其与金属和金属盐催化剂相比，避免了重金属 对环境的污染. 大量文献表明, 胺催化剂催化活性较高, 受到众多有机合成工作者的关注 ${ }^{[1 \sim 4]}$. 本文便采用了仲 胺介入的方法合成了四氢嘧啶衍生物. 很多四氢嘧啶杂 环衍生物具有良好的生物活性或药理作用, 据文献报 道, 具有该骨架的化合物对人类免疫缺陷病毒(HIV)和 阿尔兹海默病具有一定活性 ${ }^{[5,6]}$. 江焕峰课题组 ${ }^{[7 \sim 9]}$ 报道 由炔、胺和甲醛三组分在 $N, N$-二甲基甲酰胺(DMF)中一 锅法合成四氢嘧啶, 但是反应时间过长. 靳通收等 ${ }^{[10]}$ 报 道了室温无溶剂条件下, 以芳醛、 $\beta$-酮酸酯和尿素合成 四氢嘧啶酮衍生物. 胡永洲课题组 ${ }^{[11]}$ 报道了芳基异氰酸
酯与 $\beta$-芳胺基苯丙酮合成四氢嘧啶酩衍生物.

我们课题组致力于多组分反应(MCRs)构建杂环化 合物 ${ }^{[12 \sim 14]}$, 并报道了由丙炔酸甲酯和亚胺 $1 \mathrm{a}$ 一锅法合 成多取代咪唑烷的反应(Scheme 1) ${ }^{[14]}$. 在对该反应的后 续研究中, 发现仲胺可与丙炔酸甲酯顺利发生 Michael 加成反应得到缺电子烯胺 $2 \mathrm{a}$ ( $\beta$-胺基丙烯酸甲酯), 该烯 胺可与亚胺 $1 \mathrm{a}$ 在二氯乙烷中被质子酸(无水氯化氢)催 化发生反应，产生一种新的物质，对其分离后经过核磁 表征，该物质具有四氢嘧啶杂环结构(4a). 通过条件优 化, 以有机小分子羧酸代替了无水氯化氢, $\mathbf{4 a}$ 产率可达 到 $90 \%$, 并可应用于甲醛亚胺底物. 该反应具有胺基作 为离去基团的特征，从丙炔酸甲酯、亚胺到四氢嘧啶

\footnotetext{
* E-mail: yufengli@njtech.edu.cn

Received December 31, 2015; revised January 20, 2016; published online February 24, 2016.
} 


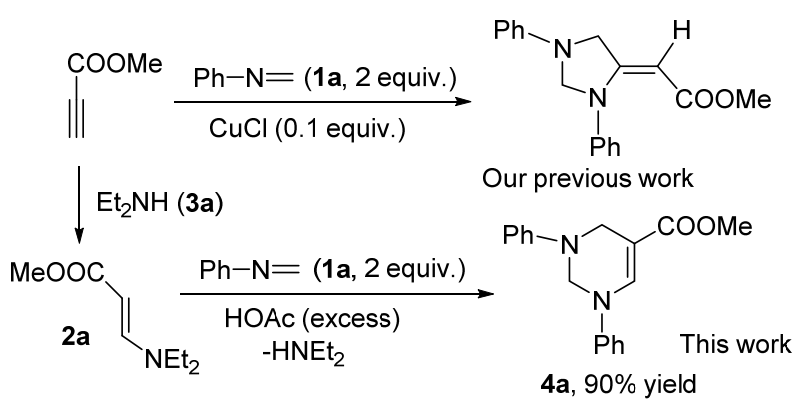

图式 1 丙炔酸甲酯与甲醛亚胺发生环合的不同途径 Scheme 1 Cyclization of methyl propiolate and imine in different routes

(4a)的转化中, 仲胺的作用类似于小分子催化剂, 对 反应方向的控制产生关键性的影响.

\section{1 结果与讨论}

\section{1 反应条件的优化}

$\beta$-胺基丙烯酸酯 2a 按照文献方法制备 ${ }^{[17,18]}$ : 二氯乙 烷作为溶剂, 二乙胺与丙炔酸甲酯顺利发生加成反应, 得到淡黄色液体 2 a.

以亚胺 ${ }^{[15,16]}(\mathbf{1 a}, 20.0 \mathrm{mmol})$ 与 $\beta$-胺基丙烯酸酯 $\mathbf{2 a}$ (10.0 mmol)为反应底物, 对反应溶剂、催化剂进行篮选, 结果见表 1 .

不使用质子酸, 仅乙醇溶剂中有产物生成, 但收率 很低，仅达到 $12 \%$ (表 1, Entry 1). 其它如 DMF、水、二 氯乙烷(DCE)、甲苯作为溶剂均无相应产物产生(表 1 , Entries 4, 7, 10 和 13). 在使用乙醇作为溶剂时, 加入等 物质的量的硫酸 $(10.0 \mathrm{mmol}$ ), 反应室温下即可进行, 并 可有效提高产物的收率至 46\% (表 1, Entry 2). 氯化氢对 反应有一定促进作用，但显著低于硫酸的催化效果 (Table 1, Entry 3). 甲苯、DMF、纯水和二氯乙烷作为溶 剂时, 硫酸的催化效果普遍优于无水氯化氢(表 1, Entries $5 \sim 6,8 \sim 9,11 \sim 12,13 \sim 15$ ). 用无机酸催化该反应 最好的结果是 DMF 作溶剂, 硫酸作催化剂, $4 \mathbf{a}$ 收率可达 到 $54 \%$.

以上结果说明质子酸对反应具有催化作用, 并且对 胺基具有成盐作用, 有利于其以铵离子形式离去. 但在 上述无机酸催化的反应中均产生大量的复杂副产物, 产 物难以结晶, 只能用层析方法进行纯化. 而使用过量甲 酸、冰醋酸有机小分子羧酸作为溶剂兼催化剂, 取得了 令人满意的结果. $2 \mathrm{a}$ 与 $1 \mathrm{a}$ 在醋酸中常温下即可迅速反 应, 仅需几分钟即可完成, 不仅反应时间大大缩短, 收 率也达到 90\% (表 1, Entry 16). 使用甲酸具有类似的效 果(表 1 , Entry 17). 反应的后处理也得到很好的优化: 反 应结束后加入适量水, 产物即可析出, 经简单重结晶即 可得到核磁级产物.
表 1 环合反应条件的优化

Table 1 Condition optimization of the cyclization

\begin{tabular}{|c|c|c|c|}
\hline $\begin{array}{c}P h \\
1 \\
N \\
\| \\
1 a(2\end{array}$ & $+{ }^{\mathrm{MeOOC}}$ & $\begin{array}{c}\text { Catalyst } \\
\text { Solvent }\end{array}$ & $\widehat{C}_{\text {COOMe }}^{N^{-P h}}$ \\
\hline Entry & Catalyst & Condition & Yield $^{a} \%$ \\
\hline 1 & - & $\mathrm{CH}_{3} \mathrm{CH}_{2} \mathrm{OH}$, Reflux, $5 \mathrm{~h}$ & 12 \\
\hline 2 & $\mathrm{H}_{2} \mathrm{SO}_{4}{ }^{b}$ & $\mathrm{CH}_{3} \mathrm{CH}_{2} \mathrm{OH}, 25{ }^{\circ} \mathrm{C}, 3 \mathrm{~h}$ & 46 \\
\hline 3 & $\mathrm{HCl}^{c}$ & $\mathrm{CH}_{3} \mathrm{CH}_{2} \mathrm{OH}, 25{ }^{\circ} \mathrm{C}, 3 \mathrm{~h}$ & 20 \\
\hline 4 & - & DMF, Reflux, 5 h & 0 \\
\hline 5 & $\mathrm{H}_{2} \mathrm{SO}_{4}{ }^{b}$ & DMF, $25{ }^{\circ} \mathrm{C}, 3 \mathrm{~h}$ & 54 \\
\hline 6 & $\mathrm{HCl}^{c}$ & $\mathrm{DMF}, 25^{\circ} \mathrm{C}, 3 \mathrm{~h}$ & 35 \\
\hline 7 & - & $\mathrm{H}_{2} \mathrm{O}$, reflux, $5 \mathrm{~h}$ & 0 \\
\hline 8 & $\mathrm{H}_{2} \mathrm{SO}_{4}{ }^{b}$ & $\mathrm{H}_{2} \mathrm{O}$, reflux, $5 \mathrm{~h}$ & 12 \\
\hline 9 & $\mathrm{HCl}^{c}$ & $\mathrm{H}_{2} \mathrm{O}$, reflux, $5 \mathrm{~h}$ & 5 \\
\hline 10 & - & DCE, reflux, $5 \mathrm{~h}$ & 0 \\
\hline 11 & $\mathrm{H}_{2} \mathrm{SO}_{4}{ }^{b}$ & DCE, $25^{\circ} \mathrm{C}, 3 \mathrm{~h}$ & 25 \\
\hline 12 & $\mathrm{HCl}^{c}$ & DCE, $25{ }^{\circ} \mathrm{C}, 3 \mathrm{~h}$ & 10 \\
\hline 13 & - & Toluene, reflux, $5 \mathrm{~h}$ & 0 \\
\hline 14 & $\mathrm{H}_{2} \mathrm{SO}_{4}{ }^{b}$ & Toluene, $25^{\circ} \mathrm{C}, 3 \mathrm{~h}$ & 30 \\
\hline 15 & $\mathrm{HCl}^{c}$ & Toluene, $25^{\circ} \mathrm{C}, 3 \mathrm{~h}$ & 11 \\
\hline 16 & $\mathrm{CH}_{3} \mathrm{COOH}^{d}$ & $\mathrm{CH}_{3} \mathrm{COOH}, 25{ }^{\circ} \mathrm{C}, 5 \mathrm{~min}$ & 90 \\
\hline 17 & $\mathrm{HCOOH}^{d}$ & $\mathrm{HCOOH}, 25^{\circ} \mathrm{C}, 5 \mathrm{~min}$ & 87 \\
\hline
\end{tabular}

\section{2 几种仲胺参与反应的情况}

对几种仲胺参与的反应进行了研究：以几种易得的 仲胺先与丙炔酸甲酯作用得到相应的 $\beta$-胺基丙烯酸甲 酯, 再以其与亚胺 1a 在醋酸存在的条件下进行环合反 应, 4a 收率情况如 Scheme 2 所示. 其中二甲胺的效果与 二乙胺的效果相当，其它杂环仲胺参与的反应收率有所 降低.
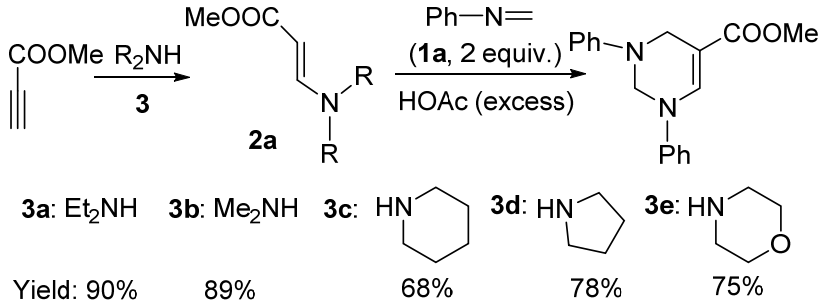

图式 2 其它仲胺参与反应的结果

Scheme 2 The results of several secondary amines-participated reactions

\section{3 亚胺底物的拓展}

在优化的反应条件下，对甲醛亚胺的结构进行了拓 展. 无论脂肪胺-甲醛亚胺(表 2, Entries 1 6) 还是芳香 胺一甲醛亚胺(表 2, Entries 7 12), 反应均获得良好的收 率. 但 $N$-亚甲基-2-吡啶胺、 $N$-亚甲基-4-氯苯胺以及 $N$ 亚甲基-3-三氟甲基苯胺按上述方法与 2a 作用均不能得 到目标产物，该结果似乎表明带有吸电子基团的芳香胺 
与甲醛形成的亚胺不适于此反应. 脂肪醛、芳香醛和糠 醛与苯胺缩合形成的亚胺也不适用于该反应. 当使用脂 肪醛-芳香胺亚胺参加反应时, 由于亚胺在反应条件下
的稳定性差，发生分解，未能得到目标产物。芳香醛和 糠醛与苯胺形成的亚胺在该反应条件下不能发生环合 反应.

表 2 四氢嘧啶衍生物的合成

Table 2 Synthesis of tetrahydropyrimidines

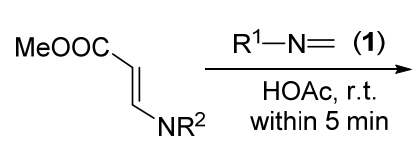

2<smiles>[R]N1C=C(C(=O)OC)CN([R])C1</smiles>

4, $75 \% \sim 95 \%$ yield

\begin{tabular}{|c|c|c|c|c|c|}
\hline Entry & Product & Yield $^{a} / \%$ & Entry & Product & Yield $^{a} / \%$ \\
\hline 1 & $\begin{array}{c}\mathrm{COC} \\
\mathbf{4 a}\end{array}$ & 90 & 7 & $4 g$ & 87 \\
\hline 2 & $4 b$ & 95 & 8 & $4 \mathrm{~h}$ & 88 \\
\hline 3 & $4 c$ & 93 & 9 & $4 i$ & 87 \\
\hline 4 & $4 d$ & 85 & 10 & & 84 \\
\hline 5 & $4 e$ & 80 & 11 & $\begin{array}{l}\text { COON } \\
\mathbf{4 k}\end{array}$ & 75 \\
\hline 6 & $\begin{array}{l}\text { COON } \\
\mathbf{4 f}\end{array}$ & 87 & 12 & $\begin{array}{l}\text { COON } \\
4 I\end{array}$ & 89 \\
\hline
\end{tabular}

${ }^{a}$ The separated yield. 


\section{4 反应机理}

根据实验结果, 该反应具有酸催化反应机理, 其可 能的历程如 Scheme 3 所示. 首先 1a 的质子化得到 5, 5 中碳正电性得到提高. $2 \mathrm{a}$ 在氮原子的给电子效应的驱动 下, 对 5 进行加成反应得到中间体 6 . 该步骤中质子酸 对 $1 \mathrm{a}$ 的质子化是非常重要的, 因为 $2 \mathrm{a}$ 中即使存在仲胺 基团的推电子作用, 酯的吸电子效应使其给出 $\pi$ 电子的 能力也是有限的. 6 对另一分子的亚胺进行亲核加成, 得到中间体 7, 7 发生分子内亲核加成得到 8 , 继而迅速 发生消去反应, 得到产物 $\mathbf{4 a}$, 同时释放出二乙铵盐. 苯 胺-芳香醛亚胺参与环合反应, 不能获得目标产物, 其 原因可能是因为芳香醛亚胺碳正电性不够, 并且位阻大 而不能接受 $\mathbf{2}$ 的加成. 带有吸电子基团的芳香胺形成的 芳香胺一甲醛亚胺参与反应未发现有任何产物产生, 我 们推测可能因为吸电子基团存在不易被醋酸质子化, 所 以反应不能引发.
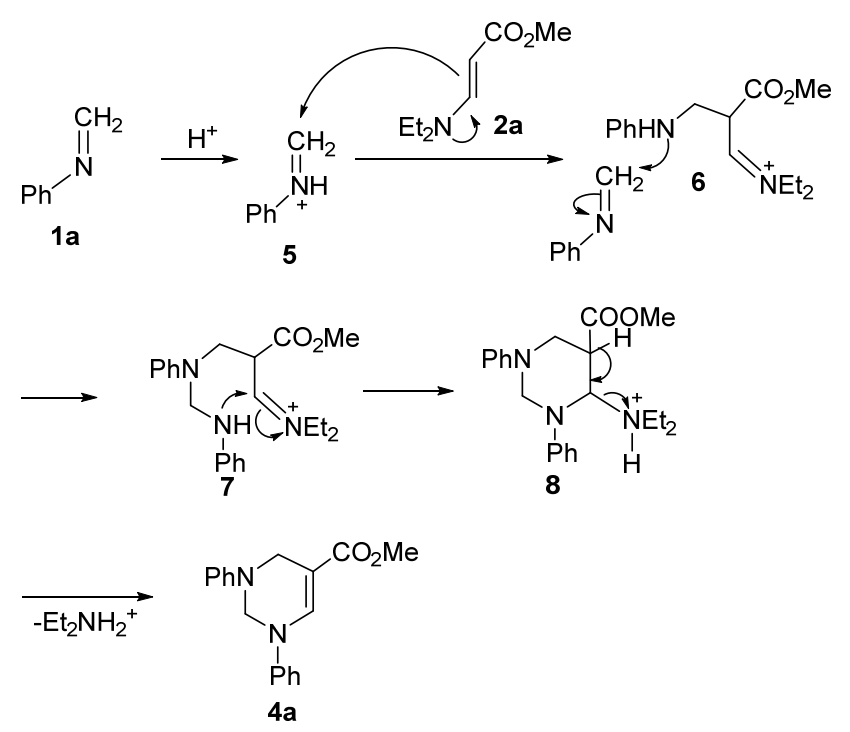

图式 3 亚胺与 $\beta$-胺基丙烯酸甲酯的环合反应历程 Scheme 3 The propbable mechanism of the cyclization between imines and $\beta$-aminoacrylate

\section{2 结论}

本文以丙炔酸甲酯为起始原料, 由小分子仲胺介 入, 先形成 $\beta$-胺基丙烯酸酯, 再与两分子亚胺在质子酸 催化下发生环合, 铵离子离去, 合成了高收率的四氢嘧 啶衍生物. 环合反应时间快, 收率高, 且后处理简单. 如 Scheme 1 所示, 丙炔酸酯与两分子亚胺的三组分反 应, 在氯化亚铜催化下可得到咪唑烷衍生物. 而由于仲 胺的介入, 则获得了结构迥异的四氢嘧啶杂环衍生物, 这正说明多组分反应往往具有可调控性. 改变反应条 件、催化剂等因素是控制其反应历程从而获得结构多样
性产物的关键.

\section{3 实验部分}

\section{1 仪器与试剂}

${ }^{1} \mathrm{H}$ NMR 和 ${ }^{13} \mathrm{C}$ NMR 用 Bruker $400 \mathrm{MHz}$ 型核磁共 振仪 $\left(\mathrm{CDCl}_{3}\right.$ 为溶剂, TMS 为内标 $)$; 熔点用 $\mathrm{X}-4$ 精密显微 熔点测定仪测定(熔点未校正); 元素分析使用的仪器为 EuroVector EA3000. 所用的药品和试剂均为市售的分 析纯或化学纯, 所有亚胺合成均参照文献 $[15,16]$, 所有 $\beta$-胺基丙烯酸甲酯合成均参照文献 $[17,18]$.

\section{2 实验方法}

\section{$3.2 .1 \beta$-二乙胺基丙烯酸甲酯(2a)的制备}

在装有电动搅拌的 $100 \mathrm{~mL}$ 四口烧瓶中加入 1,2 -二 氯乙烷 $(30 \mathrm{~mL})$ 、丙炔酸甲酯 $(0.1 \mathrm{~mol})$. 用滴液漏斗缓慢 滴加二乙胺 $(3 \mathbf{a}, 0.1 \mathrm{~mol})$, 控制反应温度 $0 \sim 5{ }^{\circ} \mathrm{C}$. 滴加 完毕后, 常温搅拌 $30 \mathrm{~min}$, TLC 跟踪至原料完全反应为 $\beta$-胺基丙烯酸酯(2a). 旋转蒸发脱去反应溶剂, 得到淡 黄色液体 2a, 产率 $97 \%$.

\subsection{2 四氢嘧啶衍生物的制备}

磁力搅拌下, 向装有醋酸 $(15 \mathrm{~mL})$ 的 $50 \mathrm{~mL}$ 四口烧 瓶中加入亚胺 $1 \mathbf{a}(20.0 \mathrm{mmol})$ 和 $\mathbf{2 a}(10.0 \mathrm{mmol})$, 常温摚 拌 $5 \mathrm{~min}$. 反应结束后加入 $70 \mathrm{~mL}$ 水，过滤，滤饼用石油 醚/乙酸乙酯混合溶剂重结晶, 烘干, 得到四氢嘧啶衍生 物 4a, 产率 90\%. 此纯化方法适用于产物 $\mathbf{4 a} \sim \mathbf{4 f}$ 的制 备.

脂肪胺一甲醛亚胺制备的四氢嘧啶衍生物 $4 \mathrm{~g} \sim 41$ 为 液体, 其纯化方法: 加入 $100 \mathrm{~mL}$ 水, 用乙酸乙酯萃取 $(20 \mathrm{~mL} \times 2)$, 减压下浓缩溶剂. 所得残余物用制备薄层 硅胶板纯化分离 $[V($ 乙酸乙酯 $): V$ (石油醚 $)=1: 10]$ 得到 最终产物。

1,3-二苯基-1,2,3,4-四氢嘧啶-5-甲酸甲酯(4a): 白色 固体, 产率 90\%. m.p. $120 \sim 122{ }^{\circ} \mathrm{C} ;{ }^{1} \mathrm{H}$ NMR $(400 \mathrm{MHz}$, $\left.\mathrm{CDCl}_{3}\right) \delta: 7.84(\mathrm{~s}, 1 \mathrm{H}), 7.38 \sim 7.34(\mathrm{~m}, 2 \mathrm{H}), 7.25 \sim 7.19$ $(\mathrm{m}, 2 \mathrm{H}), 7.12 \sim 7.09(\mathrm{~m}, 1 \mathrm{H}), 7.07 \sim 7.01(\mathrm{~m}, 2 \mathrm{H}), 6.96 \sim$ $6.86(\mathrm{~m}, 3 \mathrm{H}), 5.04(\mathrm{~s}, 2 \mathrm{H}), 4.26(\mathrm{~s}, 2 \mathrm{H}), 3.73(\mathrm{~s}, 3 \mathrm{H}) ;{ }^{13} \mathrm{C}$ NMR $\left(101 \mathrm{MHz}, \mathrm{CDCl}_{3}\right) \delta: 167.3,148.8,144.1,140.5$, 129.7, 129.3, 123.7, 121.1, 117.9, 117.8, 99.6, 77.4, 77.1, 76.8, 64.5, 51.0, 47.5. Anal. calcd for $\mathrm{C}_{18} \mathrm{H}_{18} \mathrm{~N}_{2} \mathrm{O}_{2}$ : C 73.45, H 6.16, N 9.52; found C 73.47, H 6.14, N 9.50.

1,3-二(4-甲氧基苯基)-1,2,3,4-四氢嘧啶-5-甲酸甲酯 (4b): 白色固体, 产率 95\%. m.p. $127 \sim 129{ }^{\circ} \mathrm{C} ;{ }^{1} \mathrm{H}$ NMR $\left(400 \mathrm{MHz}, \mathrm{CDCl}_{3}\right) \delta: 7.75(\mathrm{~s}, 1 \mathrm{H}), 6.99 \sim 6.84(\mathrm{~m}, 6 \mathrm{H})$, $6.80 \sim 6.74(\mathrm{~m}, 2 \mathrm{H}), 4.90(\mathrm{~s}, 2 \mathrm{H}), 4.17(\mathrm{~s}, 2 \mathrm{H}), 3.79(\mathrm{~s}$, $3 \mathrm{H}), 3.74(\mathrm{~s}, 3 \mathrm{H}), 3.71(\mathrm{~s}, 3 \mathrm{H}) ;{ }^{13} \mathrm{C}$ NMR $(101 \mathrm{MHz}$, 
$\left.\mathrm{CDCl}_{3}\right) \delta: 167.5,156.4,154.5,142.7,141.4,138.0,120.3$, 120.0, 114.9, 114.5, 97.9, 66.5, 55.5, 55.5, 50.9, 47.9. Anal. calcd for $\mathrm{C}_{20} \mathrm{H}_{22} \mathrm{~N}_{2} \mathrm{O}_{4}$ : C 67.78, H 6.26, N 7.90; found C 67.83, H 6.21, N 7.91.

1,3-二(4-甲基苯基)-1,2,3,4-四氢嘧啶-5-甲酸甲酯 (4c): 白色固体, 产率 93\%. m.p. 90 92 ${ }^{\circ} \mathrm{C} ;{ }^{1} \mathrm{H}$ NMR $\left(400 \mathrm{MHz}, \mathrm{CDCl}_{3}\right) \delta: 7.80(\mathrm{~s}, 1 \mathrm{H}), 7.15(\mathrm{~d}, J=8.3 \mathrm{~Hz}$, 2H), $7.01(\mathrm{~d}, J=8.4 \mathrm{~Hz}, 2 \mathrm{H}), 6.90$ (d, $J=8.3 \mathrm{~Hz}, 2 \mathrm{H}), 6.84$ (d, $J=8.4 \mathrm{~Hz}, 2 \mathrm{H}), 4.97$ (s, 2H), 4.22 (s, 2H), 3.71 (s, 3H), $2.32(\mathrm{~s}, 3 \mathrm{H}), 2.24(\mathrm{~s}, 3 \mathrm{H}) ;{ }^{13} \mathrm{C} \mathrm{NMR}\left(101 \mathrm{MHz}, \mathrm{CDCl}_{3}\right) \delta$ : $167.4,146.5,141.9,140.7,133.4,130.6,130.2,129.8$, 118.1, 118.0, 98.8, 65.0, 50.9, 47.7, 20.7, 20.5. Anal. calcd for $\mathrm{C}_{20} \mathrm{H}_{22} \mathrm{~N}_{2} \mathrm{O}_{2}$ : C 74.51, H 6.88, N 8.69; found C 74.48, H 6.89, N 8.68.

1,3-二(3-甲基苯基)-1,2,3,4-四氢嘧啶-5-甲酸甲酯 (4d): 白色固体, 产率 $85 \%$. m.p. $83 \sim 85{ }^{\circ} \mathrm{C}$; ${ }^{1} \mathrm{H}$ NMR $\left(400 \mathrm{MHz}, \mathrm{CDCl}_{3}\right) \delta: 7.80(\mathrm{~s}, 1 \mathrm{H}), 7.32 \sim 7.15(\mathrm{~m}, 1 \mathrm{H})$, $7.09 \sim 6.88(\mathrm{~m}, 2 \mathrm{H}), 6.84 \sim 6.63(\mathrm{~m}, 5 \mathrm{H}), 4.97(\mathrm{~s}, 2 \mathrm{H})$, $4.22(\mathrm{~s}, 2 \mathrm{H}), 3.71(\mathrm{~s}, 3 \mathrm{H}), 2.32(\mathrm{~s}, 3 \mathrm{H}), 2.24(\mathrm{~s}, 3 \mathrm{H}) ;{ }^{13} \mathrm{C}$ NMR (101 MHz, $\left.\mathrm{CDCl}_{3}\right) \delta: 167.4,148.8,144.2,140.6$, $139.7,139.0,129.5,129.1,124.5,122.0,118.9,118.6$, $114.9,114.8,99.5,64.5,51.0,47.6,21.7,21.6$. Anal. calcd for $\mathrm{C}_{20} \mathrm{H}_{22} \mathrm{~N}_{2} \mathrm{O}_{2}$ : C 74.51, H 6.88, N 8.69; found C 74.49, H 6.87, N 8.70.

1,3-二(3,5-二甲基苯基)-1,2,3,4-四氢嘧啶-5-甲酸甲 酯(4e): 黄色固体, 产率 80\%. m.p. $107 \sim 109{ }^{\circ} \mathrm{C} ;{ }^{1} \mathrm{H}$ NMR (400 MHz, $\left.\mathrm{CDCl}_{3}\right) \delta: 7.81(\mathrm{~s}, 1 \mathrm{H}), 6.74(\mathrm{~s}, 1 \mathrm{H}), 6.63$ $(\mathrm{s}, 2 \mathrm{H}), 6.55 \sim 6.53(\mathrm{~m}, 3 \mathrm{H}), 4.94(\mathrm{~s}, 2 \mathrm{H}), 4.20(\mathrm{~s}, 2 \mathrm{H})$, $3.72(\mathrm{~s}, 3 \mathrm{H}), 2.31(\mathrm{~s}, 6 \mathrm{H}), 2.20(\mathrm{~s}, 6 \mathrm{H}) ;{ }^{13} \mathrm{C}$ NMR $(101$ $\left.\mathrm{MHz}, \mathrm{CDCl}_{3}\right) \delta: 167.4,148.9,144.3,140.8,139.4$, $138.7,125.5,122.9,115.9,115.8,99.3,64.6,50.9,47.6$, 21.6, 21.5. Anal. calcd for $\mathrm{C}_{22} \mathrm{H}_{26} \mathrm{~N}_{2} \mathrm{O}_{2}$ : C 75.40, H 7.48, N 7.99; found C 75.39, H 7.46, N 8.00.

1,3-二(3-叔丁基苯基)-1,2,3,4-四氢嘧啶-5-甲酸甲酯 (4f): 黄色固体, 产率 87\%. m.p. 97 99 ${ }^{\circ} \mathrm{C} ;{ }^{1} \mathrm{H} \mathrm{NMR}$ $\left(400 \mathrm{MHz}, \mathrm{CDCl}_{3}\right) \delta: 7.73(\mathrm{~s}, 1 \mathrm{H}), 7.15 \sim 7.13(\mathrm{~m}, 2 \mathrm{H})$, $7.02 \sim 6.92(\mathrm{~m}, 2 \mathrm{H}), 6.89 \sim 6.87(\mathrm{~m}, 2 \mathrm{H}), 6.82 \sim 6.63(\mathrm{~m}$, 2H), 4.92 (s, 2H), 4.19 (s, 2H), 3.58 (s, 3H), 1.20 (s, 9H), $1.02(\mathrm{~s}, 9 \mathrm{H}) ;{ }^{13} \mathrm{C}$ NMR (101 MHz, $\left.\mathrm{CDCl}_{3}\right) \delta: 167.4,153.2$, $152.3,148.8,144.2,140.6,129.4,128.9,121.0,118.3$, $115.6,115.3,115.1,114.9,99.8,64.6,50.9,48.5,34.9$, 34.7, 31.4, 31.3. Anal. calcd for $\mathrm{C}_{26} \mathrm{H}_{34} \mathrm{~N}_{2} \mathrm{O}_{2}$ : C 76.81, $\mathrm{H}$ 8.43, N 6.89; found C 76.83, H 8.46, N 6.87.

1,3-二正丁基-1,2,3,4-四氢嘧啶-5-甲酸甲酯(4g): 黄 色液体, 产率 $87 \%$. ${ }^{1} \mathrm{H}$ NMR $\left(400 \mathrm{MHz}, \mathrm{CDCl}_{3}\right) \delta: 7.38$ (s, 1H), 3.90 (s, 2H), 3.65 (s, 3H), $3.46(\mathrm{~s}, 2 \mathrm{H}), 3.11 \sim 3.08$ $(\mathrm{m}, 2 \mathrm{H}), 2.51 \sim 2.45(\mathrm{~m}, 2 \mathrm{H}), 1.56 \sim 1.45(\mathrm{~m}, 4 \mathrm{H}), 1.41 \sim$ $1.24(\mathrm{~m}, 4 \mathrm{H}), 0.95$ (t, $J=8.8 \mathrm{~Hz}, 3 \mathrm{H}), 0.92(\mathrm{t}, J=8.5 \mathrm{~Hz}$, $3 \mathrm{H}) ;{ }^{13} \mathrm{C}$ NMR (101 MHz, $\left.\mathrm{CDCl}_{3}\right) \delta: 167.9,144.7,91.5$, 66.6, 53.6, 52.6, 50.3, 47.7, 31.1, 30.0, 20.5, 19.7, 13.9, 13.6. Anal. calcd for $\mathrm{C}_{14} \mathrm{H}_{26} \mathrm{~N}_{2} \mathrm{O}_{2}$ : C 66.10, $\mathrm{H} 10.30, \mathrm{~N}$ 11.01; found C 66.08, H 10.28, N 11.04.

1,3-二苄基-1,2,3,4-四氢嘧啶-5-甲酸甲酯(4h)：黄色 固体, 产率 $88 \%$. m.p. 82 $84{ }^{\circ} \mathrm{C} ;{ }^{1} \mathrm{H}$ NMR $(400 \mathrm{MHz}$, $\left.\mathrm{CDCl}_{3}\right) \delta: 7.62(\mathrm{~s}, 1 \mathrm{H}), 7.33 \sim 7.30(\mathrm{~m}, 3 \mathrm{H}), 7.29 \sim 7.24$ $(\mathrm{m}, 2 \mathrm{H}), 7.23 \sim 7.17(\mathrm{~m}, 5 \mathrm{H}), 4.20(\mathrm{~s}, 2 \mathrm{H}), 3.81(\mathrm{~s}, 2 \mathrm{H})$, 3.67 (s, 3H), 3.57 (s, 2H), 3.53 (s, 2H); ${ }^{13} \mathrm{C}$ NMR (101 $\left.\mathrm{MHz}, \mathrm{CDCl}_{3}\right) \delta: 168.1,144.9,138.3,136.7,128.9,128.3$, $128.0,127.7,127.2,93.0,65.4,57.7,57.2,50.6,47.9$. Anal. calcd for $\mathrm{C}_{20} \mathrm{H}_{22} \mathrm{~N}_{2} \mathrm{O}_{2}$ : C 74.51, H 6.88, N 8.69; found $\mathrm{C} 74.52, \mathrm{H} 6.89, \mathrm{~N} 8.70$.

1,3-二环己基-1,2,3,4-四氢嘧啶-5-甲酸甲酯(4i)：黄 色液体, 产率 $87 \% .{ }^{1} \mathrm{H}$ NMR $\left(400 \mathrm{MHz}, \mathrm{CDCl}_{3}\right) \delta: 7.48$ $(\mathrm{s}, 1 \mathrm{H}), 4.04(\mathrm{~s}, 2 \mathrm{H}), 3.64(\mathrm{~s}, 3 \mathrm{H}), 3.55(\mathrm{~s}, 2 \mathrm{H}), 2.95 \sim 2.85$ $(\mathrm{m}, 1 \mathrm{H}), 2.50 \sim 2.40(\mathrm{~m}, 1 \mathrm{H}), 1.99 \sim 1.88(\mathrm{~m}, 2 \mathrm{H}), 1.88 \sim$ $1.72(\mathrm{~m}, 5 \mathrm{H}), 1.72 \sim 1.66(\mathrm{~m}, 3 \mathrm{H}), 1.51 \sim 1.45(\mathrm{~m}, 2 \mathrm{H})$, $1.45 \sim 1.35(\mathrm{~m}, 5 \mathrm{H}), 1.35 \sim 1.06(\mathrm{~m}, 3 \mathrm{H}) ;{ }^{13} \mathrm{C}$ NMR $(101$ $\left.\mathrm{MHz}, \mathrm{CDCl}_{3}\right) \delta: 167.7,143.3,92.6,62.4,62.1,58.3,50.2$, $44.9,31.8,30.5,25.9,25.6,25.4,25.2$. Anal. calcd for $\mathrm{C}_{18} \mathrm{H}_{30} \mathrm{~N}_{2} \mathrm{O}_{2}$ : C 70.55, H 9.87, N 9.14; found $\mathrm{C} 70.52, \mathrm{H}$ 9.87, N 9.16 .

1,3-二乙基-1,2,3,4-四氢嘧啶-5-甲酸甲酯(4j): 黄色 液体, 产率 $84 \% .{ }^{1} \mathrm{H}$ NMR $\left(400 \mathrm{MHz}, \mathrm{CDCl}_{3}\right) \delta: 7.40(\mathrm{~s}$, $1 \mathrm{H}), 3.92$ (s, 2H), 3.64 (s,3H), 3.44 (s, 2H), 3.17 (q, $J=7.2$ $\mathrm{Hz}, 2 \mathrm{H}), 2.55$ (q, $J=7.2 \mathrm{~Hz}, 2 \mathrm{H}), 1.18$ (t, $J=7.2 \mathrm{~Hz}, 3 \mathrm{H})$, $1.14(\mathrm{t}, J=7.2 \mathrm{~Hz}, 3 \mathrm{H}) ;{ }^{13} \mathrm{C} \mathrm{NMR}\left(101 \mathrm{MHz}, \mathrm{CDCl}_{3}\right) \delta$ : 167.7, 144.0, 91.8, 65.8, 50.2, 48.0, 47.2, 46.8, 14.2, 13.0. Anal. calcd for $\mathrm{C}_{10} \mathrm{H}_{18} \mathrm{~N}_{2} \mathrm{O}_{2}$ : C 60.58, $\mathrm{H}$ 9.15, N 14.13; found $\mathrm{C} 60.70, \mathrm{H} 9.16, \mathrm{~N} 14.13$.

1,3-二丙基-1,2,3,4-四氢嘧啶-5-甲酸甲酯(4k): 黄色 液体, 产率 75\%. ${ }^{1} \mathrm{H}$ NMR (400 $\left.\mathrm{MHz}, \mathrm{CDCl}_{3}\right) \delta: 7.13$ (s, $1 \mathrm{H}), 3.64$ (s, 2H), 3.37 (s, 3H), 3.17 (s, 2H), 2.80 (t, $J=7.1$ $\mathrm{Hz}, 2 \mathrm{H}), 2.20$ (t, $J=7.2 \mathrm{~Hz}, 2 \mathrm{H}), 1.33 \sim 1.23(\mathrm{~m}, 4 \mathrm{H}), 0.66$ $(\mathrm{t}, J=7.1 \mathrm{~Hz}, 3 \mathrm{H}), 0.65(\mathrm{t}, J=7.2 \mathrm{~Hz}, 3 \mathrm{H}) ;{ }^{13} \mathrm{C} \mathrm{NMR}(101$ $\left.\mathrm{MHz} \mathrm{CDCl}_{3}\right) \delta: 167.6,144.5,91.3,66.5,55.3,54.6,49.9$, 47.4, 22.1, 20.9, 11.5, 10.8. Anal. calcd for $\mathrm{C}_{12} \mathrm{H}_{22} \mathrm{~N}_{2} \mathrm{O}_{2}$ : C 63.68, H 9.80, N 12.38; found C 63.68, H 9.81, N 12.36.

1,3-二异丙基-1,2,3,4-四氢嘧啶-5-甲酸甲酯(4I): 黄 色液体, 产率 $89 \% .{ }^{1} \mathrm{H}$ NMR $\left(400 \mathrm{MHz}, \mathrm{CDCl}_{3}\right) \delta: 7.47$ (s, 1H), 3.95 (s, 2H), 3.66 (s, 3H), 3.50 (s, 2H), 3.46 3.32 
(m, 1H), 2.90 2.83 (m, 1H), $1.23(\mathrm{~s}, 3 \mathrm{H}), 1.22(\mathrm{~s}, 3 \mathrm{H})$, $1.15(\mathrm{~s}, 3 \mathrm{H}), 1.13(\mathrm{~s}, 3 \mathrm{H}) ;{ }^{13} \mathrm{C}$ NMR (101 MHz, $\left.\mathrm{CDCl}_{3}\right) \delta$ : 167.6, 142.7, 93.2, 62.0, 53.7, 50.3, 50.2, 45.1, 21.1, 20.1. Anal. calcd for $\mathrm{C}_{12} \mathrm{H}_{22} \mathrm{~N}_{2} \mathrm{O}_{2}$ : C 63.68, H 9.80, N 12.38; found $\mathrm{C} 63.68, \mathrm{H} 9.81, \mathrm{~N} 12.37$.

辅助材料(Supporting Information) 化合物 $\mathbf{4 a} \sim 4 \mathbf{i}$ 的 核磁共振氢谱和碳谱. 这些材料可以免费从本刊网站 (http://sioc-journal.cn/)上下载.

\section{References}

[1] Catherine, A.; Scott, J. J. Am. Chem. Soc. 2003, 125, 12394.

[2] Varinder, K.; Chrystel, L.; Franck S. J. Am. Chem. Soc. 2003, 125, 7596.

[3] Morris, M.; Michael, M.; Bernd, S.; Rainer, M. J. Am. Chem. Soc. 2007, 129, 7258

[4] Kerstin, R.; Rainer, M. Org. Lett. 2011, 13, 1878.

[5] De Lucca, G.; Liang, J.; De Lucca, I. J. Med. Chem. 1999, 42, 135.

[6] Dunbar, P.; Durant, G.; Rho, T.; Ojo, B.; Huzl, J.; Smith, A.; Sbeih, S.; Ngur, D.; Periyasamy, S.; Hoss, W.; Messer, W. J. Med. Chem. 1994, 37, 2774.

[7] Cao, H.; Wang, X.; Jiang, H.; Zhu, Q.; Zhang, M.; Liu, H. Chem.
Eur. J. 2008, 14, 11623.

[8] Zhang, M.; Jiang, H.; Liu, H.; Zhu, Q. Org. Lett. 2007, 9, 4111.

[9] Zhu, Q.; Jiang, H.; Li, J.; Zhang, M.; Wang, X. Tetrahedron 2009, $65,4604$.

[10] Jin, T.; Zhao, Y.; Liu, L.; Li, T. Chin. J. Org. Chem.2006, 26, 975 (in Chinese).

(靳通收，赵芗，刘利宾，李同双，有机化学, 2006, 26, 975.)

[11] Xue, N.; Chen, Y.; Lv, X.; Hu, Y. Chin. J. Org. Chem. 2008, 28, 325 (in Chinese).

(薛娜, 陈也伟, 吕秀阳, 胡永洲, 有机化学, 2008, 28, 325.)

[12] Li, Y.; Shi, J.; Wu, Z.; Wang, X.; Wu, X.; Gu, J.; Bu, H.; Ma, H. Tetrahedron 2014, 70, 2472.

[13] Li, Y.; Wu, Z.; Shi, J.; Pan, Y.; Bu, H.; Ma, H.; Gu, J.; Huang, H.; Wang, Y.; Wu, L. Tetrahedron 2014, 70, 8971.

[14] Li, Y.; Wu, Z.; Shi, J.; Bu, H.; Gu, J.; Pan, Y. Tetrahedron 2014, 70, 3134.

[15] Sartori, G.; Bigi, F.; Maggi, R. Eur. J. Org. Chem. 2007, 72, 1315.

[16] Malkov, A.; Vrankova, K.; Stoncius, S. J. Org. Chem. 2009, 74, 5839.

[17] Jiang, Y.; Zhong, Y.; Lourdusamy, E.; Park, C. Chem. Commun. 2012, 48, 3133.

[18] Drev, M.; Groselj, U.; Mevec, S.; Pusavec, E.; Strekelj, J.; Golobic, A.; Dahmann, G.; Stanovnik, B.; Svete, J. Tetrahedron 2014, 70, 8267. 